\title{
Study on Moderation Properties of Cold Mesitylene using KUANS
}

\author{
Seiji Tasaki ${ }^{1, *}$, Yuma Idobata $^{1}$, Yuya Adachi ${ }^{1}$, Fumiaki Funama $^{1}$, and Yutaka Abe ${ }^{1}$ \\ ${ }^{1}$ Department of Nuclear Engineering, Kyoto University, Kyotodaigaku-Katsura Nishikyou-ku Kyoto 615-8530, Japan
}

\begin{abstract}
Neutron moderation properties from the cold mesitylene moderator have been studied. Kyoto University Accelerator driven Neutron Source has been used for these experiments. The container of the mesitylene moderator is situated in front of the polyethylene moderator and the change of the time of flight spectrum has been recorded as a function of the temperature of the mesitylene moderator. By fitting the Maxwell distribution to the obtained TOF spectra, the neutron temperature corresponding to the mesitylene temperature is estimated.
\end{abstract}

\section{Introduction}

In recent years, compact neuron sources have been developed. In spite of their low neutron flux, they may be useful by specific scientific domains. For study of nanoscale structure in materials using small angle scattering, or reflectometry, cold neutrons are commonly used because the separation between the incident and the diffracted neutron beams are roughly proportional to the wavelength and larger and easier to measure with cold neutrons.

For producing such long-wavelength neutrons, cold neutron sources (CNS) are employed. For most of such CNS, liquid hydrogen or solid $\mathrm{CH}_{4}$ are used as cold moderator. For compact sources, however, such materials are not suitable because a) they are gas in room temperature and the volume change is quite large when they become liquid or solid, and b) they are explosive in the gas state. Mesitylene having three metyl's around the benzene ring and staying liquid over a large range of temperatures has been suggested as a good moderator for small neutron sources [1].

In the present study, we measured the wavelength distribution from the cooled mesitylene moderator in order to clarify the moderation properties for neutrons of cold mesitylene using Kyoto University Acceleratordriven Neutron Source (KUANS).

In KUANS neutrons are produced by ${ }^{9} \mathrm{Be}(\mathrm{p}, \mathrm{n}){ }^{9} \mathrm{~B}$ reaction using pulsed $3.5 \mathrm{MeV}$-proton beam. The neutrons are moderated by the polyethylene and the moderated neutrons are emitted to the direction perpendicular to the proton beam [1].

\section{Measurements}

Figure 1 shows schematic view of the experimental set-up for the present measurements.
The mesitylene moderator is situated in front of the polyethylene moderator having the size of $120 \times 120 \times 85$ $\mathrm{mm}^{3}$ in room temperature. The mesitylene container with $25(\mathrm{t}) \times 96(\mathrm{w}) \times 100(\mathrm{~h}) \mathrm{mm}^{3}$ is held in a vacuum container with the size of $43(\mathrm{t}) \times 123(\mathrm{w}) \times 155(\mathrm{~h}) \mathrm{mm}^{3}$. The moderators are surrounded by the graphite reflector.

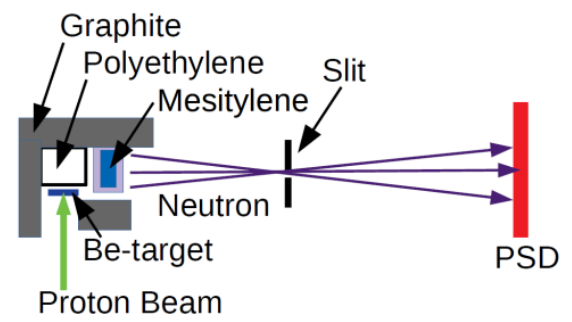

Fig. 1. Experimental set-up of the present study.

The neutrons emitted from the moderator surface go through the $20(\mathrm{~h}) \times 5(\mathrm{w}) \mathrm{mm}^{2}$-slit situated at $1710 \mathrm{~mm}$ from the moderator surface and are detected with $3 \mathrm{He} 1$ dimensional position sensitive detector (PSD) at $1546 \mathrm{~mm}$ from the slit. With the PSD, it is possible to measure 1dimensional 'pin hole image' over the moderator as well as the time of flight (TOF) spectrum. The TOF spectra presented in this report are taken from the mesitylene area of the PSD data. Total flight length is $3256 \mathrm{~mm}$ and T0 (time between the system trigger and emission of thermal neutron from the moderator) is $70 \mu$ s for KUANS. The repetition rate and pulse width of the proton pulse are $100 \mathrm{~Hz}$ and $60 \mu \mathrm{s}$, respectively. Average proton current was about $60 \mu \mathrm{A}$. Since the time bin for TOF measurements is $10 \mu \mathrm{s}, 6$ successive channels of TOF data are arithmetically averaged.

Mesitylene moderator was cooled with cryostat from room temperature to $28 \mathrm{~K}$. During cooling process (about $10 \mathrm{hr}$ ), the accelerator was operated, and the neutron spectra were measured. The TOF data are summed up for

\footnotetext{
* Corresponding author: tasaki@nucleng.kyoto-u.ac.jp
} 
every 30min during when the temperature changes about 10 degree in kelvin. We adopt mean temperature for each TOF data.

The TOF spectra of neutron from the mesitylene moderator for the mesitylene temperature $T_{\mathrm{M}}=251,204$, 141, 84, 28K are shown in Fig. 2. Change of the spectrum is small for over $200 \mathrm{~K}$ which is close to the melting point of mesitylene. As the $T_{\mathrm{M}}$ decreased, the peak intensity is lowered and shifted to the longer wavelength side.

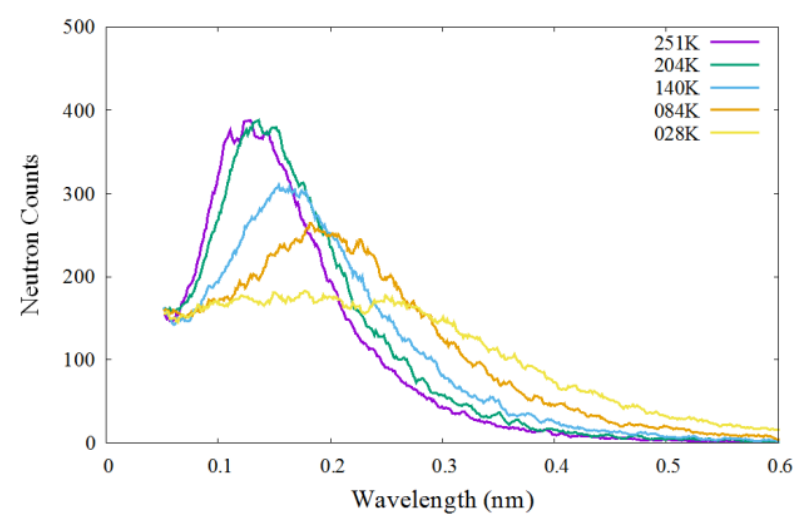

Fig. 2. TOF neutron spectra for various temperature of the mesitylene moderator.

The relative neutron intensity versus wavelength for $T_{\mathrm{M}}=$ $204,141,84,28 \mathrm{~K}$ comparing to that for $251 \mathrm{~K}$ is shown in Fig. 3.

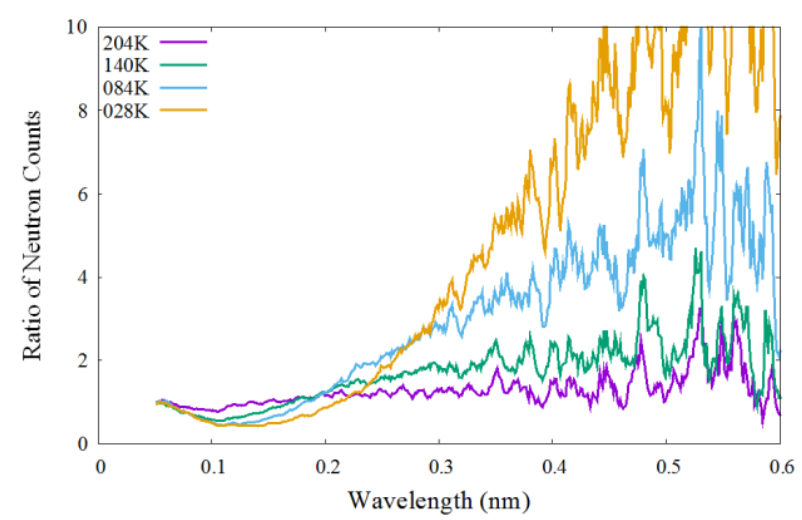

Fig. 3. Relative neutron intensity for some temperature comparing to that for $251 \mathrm{~K}$.

For wavelength longer than $0.25 \mathrm{~nm}$, the ratio is larger than unity and increases as the wavelength becomes longer and as $T_{\mathrm{M}}$ lowers. At the lowest temperature of $28 \mathrm{~K}$ and at wavelength of $0.4 \mathrm{~nm}$, neutron intensity is six times higher compard to that at $251 \mathrm{~K}$.

\section{Discussion}

In order to obtain the neutron temperature, we fit a Maxwell distribution to the neutron wavelength distribution shown in Fig.2. The aim of the fit is to obtain the temperature of the neutrons $T_{\mathrm{n}}$ in the cold mesitylene moderator. Hence the fitting is carried out for neutrons with wavelength longer than $0.2 \mathrm{~nm}$. Examples of the fit results are shown in Fig.4. The fit function is the neutron flux and has the following form for neutron wavelength of $\lambda$ :

$$
\phi(\lambda)=\frac{A}{\lambda^{5}} \exp \left[-\frac{T_{0}}{T_{n}}\left(\frac{\lambda_{0}^{2}}{\lambda^{2}}\right)\right]
$$

where $\lambda_{0}=0.178 \mathrm{~nm}$, and $T_{0}=300 \mathrm{~K}$ are the wavelength and corresponding temperature for $25 \mathrm{meV}$-neutron. The fit parameters are $A$ and $T_{\mathrm{n}}$, the latter of which represents the moderated neutron temperature.

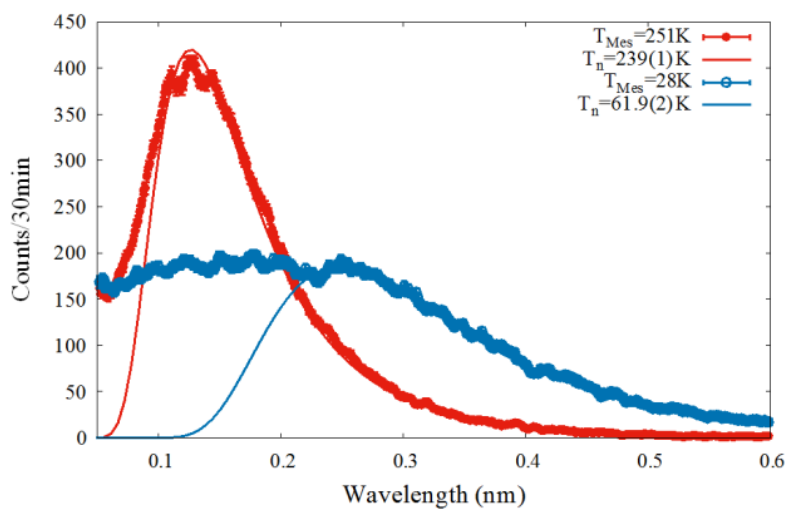

Fig. 4. Examples of Maxwellian fit to neutron TOF data shown in Fig.2 in the $251 \mathrm{~K}$ (red) and $28 \mathrm{~K}$ (blue) case.

The red and blue line in Fig.4 represent the spectrum for $T_{\mathrm{M}}=251 \mathrm{~K}$ and $28 \mathrm{~K}$, respectively. For the former case $T_{\mathrm{n}}=239 \mathrm{~K}$, and for the latter $T_{\mathrm{n}}=61.9 \mathrm{~K}$, respectively. There may be two reasons why $T_{\mathrm{n}}$ is higher than $T_{\mathrm{M}}$ : (1) Because the number of excitation levels around several meV range in mesitylene is not enough, some neutrons are emitted before being fully moderated. (2) Thickness $25 \mathrm{~mm}$ of the mesitylene moderator container is not enough for full moderation of neutron. Hence the discrepancy between the fit function and TOF spectrum in the short wavelength range is due to the under-moderation of the neutrons.

Repeating the same procedure for other temperature of mesitylene, the relation between $T_{\mathrm{M}}$ and $T_{\mathrm{n}}$ has been obtained. The result is shown in Fig.6.

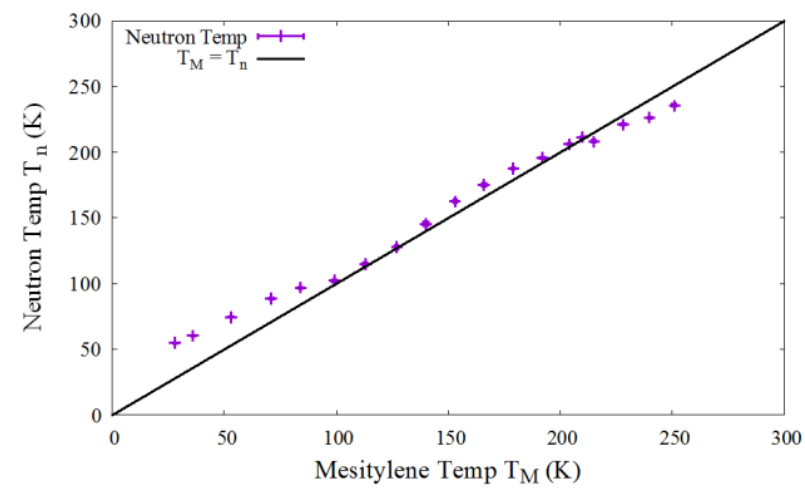

Fig. 6. The relation between $T_{\mathrm{M}}$ and $T_{\mathrm{n}}$.

In the figure, the solid line stands for the relation $T_{\mathrm{M}}=T_{\mathrm{n}}$. When $T_{\mathrm{M}}>100 \mathrm{~K}, T_{\mathrm{n}}$ changes along $T_{\mathrm{M}}$, which means that the neutron temperature follows the mesitylene temperature. 
On the other hand, when $T_{\mathrm{M}}<100 \mathrm{~K}$ there appears discrepancy between $T_{\mathrm{M}}$ and $T_{\mathrm{n}}$, which means that at low mesitylene temperature neutrons are under-moderated; their temperature stays above the mesitylene temperature. In spite that, cooled mesitylene enables us to obtain an increase of flux of long wavelength neutrons with the factor 10 at $0.5 \mathrm{~nm}$ compared to ambient temperature.

The slowing down distance for mesitylene is estimated to be about $6 \mathrm{~cm}$, since that for $\mathrm{H}_{2} \mathrm{O}$ is about $5 \mathrm{~cm}$ and is reverse proportional to the hydrogen density $\left(6.7 \times 10^{22}\right.$, 5.19 $\times 10^{22}$ for $\mathrm{H}_{2} \mathrm{O}$ and mesitylene, respectively) [3]. The size of the mesitylene container is $25 \times 96 \times 100 \mathrm{~mm}^{3}$ in the present experiments, and the 'thickness' $25 \mathrm{~mm}$ is too short for fully moderation. The neutron flux may be improved if the neutron is emitted the 'side' of the container.

\section{Conclusions}

Neutron TOF spectra from the mesitylene moderator cooled from the room temperature to $28 \mathrm{~K}$ were measured. Measured spectra have changed corresponding to the mesitylene temperature. As the mesitylene temperature decreases, the peak of the spectrum shifts to longer wavelength side, and the neutron counts in the wavelength range longer than $0.2 \mathrm{~nm}$ increases. At $0.4 \mathrm{~nm}$, the ratio of the neutron counts from the cooled mesitylene against uncooled mesitylene becomes about 6 . The neutron counts with the wavelength over $0.2 \mathrm{~nm}$, is well reproduced by the Maxwellian distribution. From the fit, neutron temperature is obtained and is almost the same in the temperature range above $100 \mathrm{~K}$, and the difference appears under $100 \mathrm{~K}$. The neutron flux may be improved by the optimization of the arrangement of the moderator system.

\section{Acknowledgement}

We would like to thank Mr. M. Hirose and Prof. T. Nagae for the operation of KUANS and the mesitylene moderator.

\section{References}

1. Lourdes Torres, J.R. Granada, Physica B: Condensed Matter, 385-386, Part 2, 2006, pp 1306-1308.

2. S. Tasaki et al., proceedings of Ucans III\&IV, Physics Procedia 60, 2014, pp 181-185.

3. J. R. Lamarsh, Introduction to Nuclear Reactor Theory, Addison-Wesley Publishing Company Inc., Massachusetts, USA 1965. 